\title{
ON FIXED POINTS OF NONEXPANSIVE MAPPINGS IN NONCONVEX SETS
}

\author{
W. G. DOTSON, JR.
}

\begin{abstract}
Two theorems are proved concerning the existence of fixed points of nonexpansive mappings on a certain class of nonconvex sets. This work extends the author's previous work on starshaped sets.
\end{abstract}

Suppose $S$ is a subset of a Banach space $E$, and let $F=\left\{f_{\alpha}\right\}_{\alpha \in S}$ be a family of functions from $[0,1]$ into $S$, having the property that for each $\alpha \in S$ we have $f_{\alpha}(1)=\alpha$. Such a family $F$ is said to be contractive provided there exists a function $\phi:(0,1) \rightarrow(0,1)$ such that for all $\alpha$ and $\beta$ in $S$ and for all $t$ in $(0,1)$ we have

$$
\left\|f_{\alpha}(t)-f_{\beta}(t)\right\| \leqq \phi(t)\|\alpha-\beta\| .
$$

Such a family $F$ is said to be jointly continuous provided that if $t \rightarrow t_{0}$ in $[0,1]$ and $\alpha \rightarrow \alpha_{0}$ in $S$ then $f_{\alpha}(t) \rightarrow f_{\alpha_{0}}\left(t_{0}\right)$ in $S$.

THEOREM 1. Suppose $S$ is a compact subset of a Banach space E, and suppose there exists a contractive, jointly continuous family $F$ of functions associated with $S$ as described above. Then any nonexpansive self-mapping $T$ of $S$ has a fixed point in $S$.

Proof. For each $n=1,2,3, \cdots$, let $k_{n}=n /(n+1)$, and let $T_{n}: S \rightarrow S$ be defined by $T_{n} x=f_{T x}\left(k_{n}\right)$ for all $x \in S$. Since $T(S) \subset S$ and $0<k_{n}<1$, we have that each $T_{n}$ is well-defined and maps $S$ into $S$. Furthermore, for each $n$ we have, for all $x, y$ in $S$,

$\left\|T_{n} x-T_{n} y\right\|=\left\|f_{T x}\left(k_{n}\right)-f_{T y}\left(k_{n}\right)\right\| \leqq \phi\left(k_{n}\right)\|T x-T y\| \leqq \phi\left(k_{n}\right)\|x-y\|$,

so that, for each $n, T_{n}$ is a contraction mapping on $S$. As a compact (hence closed) subset of the Banach space $E, S$ is a complete metric space. Therefore each $T_{n}$ has a unique fixed point $x_{n} \in S$. Since $S$ is compact, there is a subsequence $\left\{x_{n_{j}}\right\}$ of $\left\{x_{n}\right\}$ such that $x_{n_{j}} \rightarrow$ some $x \in S$. Since $T_{n_{j}} x_{n_{j}}=x_{n_{j}}$ we

Received by the editors June 20, 1972.

AMS (MOS) subject classifications (1970). Primary 47H10; Secondary 47H99.

Key words and phrases. Nonexpansive mappings, fixed points, star-shaped sets.

(c) American Mathematical Society 1973 
have $T_{n_{j}} x_{n_{j}} \rightarrow x$. But $T$ is continuous (since nonexpansive), and so $T x_{n_{i}} \rightarrow$ $T x$. The joint continuity now yields

$$
T_{n_{j}} x_{n_{j}}=f_{T x_{n_{j}}}\left(k_{n_{j}}\right) \rightarrow f_{T x}(1)=T x .
$$

It follows that $T x=x$, since $E$ is Hausdorff. Q.E.D.

A special case of the above theorem is Theorem 1 of [1], where $S$ is assumed to be star-shaped. With $p$ a star-center and $k_{n}=n /(n+1)$ we have $f_{\alpha}(t)=(1-t) p+t \alpha$ so that $T_{n} x=f_{T x}\left(k_{n}\right)=\left(1-k_{n}\right) p+k_{n} T x$. One easily checks that

$$
\left\|f_{\alpha}(t)-f_{\beta}(t)\right\| \leqq t\|\alpha-\beta\|
$$

so that we can take $\phi(t)=t$ for $0<t<1$; and it is a well-known fact that $f_{\alpha}(t)=(1-t) p+t \alpha$ is jointly continuous in $t$ and $\alpha$.

A family $F=\left\{f_{\alpha}\right\}_{\alpha \in S}$ of functions from $[0,1]$ into a set $S$ will be called jointly weakly continuous provided that if $t \rightarrow t_{0}$ in $[0,1]$ and $\alpha \rightarrow \alpha_{0}$ in $S$ then $f_{\alpha}(t) \rightarrow f_{\alpha_{0}}\left(t_{0}\right)$ in $S$ (here $\rightarrow$ denotes weak convergence).

THEOREM 2. Suppose $S$ is a weakly compact subset of a Banach space $E$, and suppose there exists a contractive, jointly weakly continuous family $F$ of functions associated with $S$ as described above and before Theorem 1. Then any nonexpansive weakly continuous self-mapping $T$ of $S$ has a fixed point in $S$.

Proof. As in Theorem 1 , let $k_{n}=n /(n+1)$ and define $T_{n}: S \rightarrow S$ by $T_{n} x=f_{T x}\left(k_{n}\right)$ for all $x \in S$ and for all $n=1,2,3, \cdots$. Then, as before, each $T_{n}$ is a contraction mapping on $S$. Since the weak topology of $E$ is Hausdorff and $S$ is weakly compact, we have that $S$ is weakly closed and therefore strongly closed. Hence $S$ is a complete metric space (with the norm topology of the Banach space $E$ ), and so each $T_{n}$ has a unique fixed point $x_{n} \in S$. By the Eberlein-Smulian theorem $S$ is weakly sequentially compact. Thus there is a subsequence $\left\{x_{n_{j}}\right\}$ of $\left\{x_{n}\right\}$ such that $x_{n_{j}} \rightarrow$ some $x \in S$. Since $T_{n_{j}} x_{n_{j}}=x_{n_{j}}$ we have $T_{n_{j}} x_{n_{j}} \rightarrow x$. Since $T$ is weakly continuous we have $T x_{n_{j}} \rightarrow T x$. The joint weak continuity now yields $T_{n_{j}} x_{n_{j}}=$ $f_{T x_{n_{j}}}\left(k_{n_{j}}\right) \rightarrow f_{T x}(1)=T x$. Since the weak topology is Hausdorff, we now get $T x=x$. Q.E.D.

\section{REFERENCE}

1. W. G. Dotson, Jr., Fixed point theorems for nonexpansive mappings on starshaped subsets of Banach spaces, J. London Math. Soc. (2) 4 (1972), 408-410.

Department of Mathematics, North Carolina State University at Raleigh, Raleigh, North Carolina 27607 